\title{
Review Article \\ Fuzzy Stochastic Differential Equations Driven by Semimartingales-Different Approaches
}

\author{
Mariusz Michta \\ Faculty of Mathematics, Computer Science and Econometrics, University of Zielona Góra, Szafrana 4a, 65-516 Zielona Góra, Poland \\ Correspondence should be addressed to Mariusz Michta; m.michta@wmie.uz.zgora.pl
}

Received 17 December 2014; Accepted 16 February 2015

Academic Editor: Elena Benvenuti

Copyright ( 2015 Mariusz Michta. This is an open access article distributed under the Creative Commons Attribution License, which permits unrestricted use, distribution, and reproduction in any medium, provided the original work is properly cited.

\begin{abstract}
The first aim of the paper is to present a survey of possible approaches for the study of fuzzy stochastic differential or integral equations. They are stochastic counterparts of classical approaches known from the theory of deterministic fuzzy differential equations. For our aims we present first a notion of fuzzy stochastic integral with a semimartingale integrator and its main properties. Next we focus on different approaches for fuzzy stochastic differential equations. We present the existence of fuzzy solutions to such equations as well as their main properties. In the first approach we treat the fuzzy equation as an abstract relation in the metric space of fuzzy sets over the space of square integrable random vectors. In the second one the equation is interpreted as a system of stochastic inclusions. Finally, in the last section we discuss fuzzy stochastic integral equations with solutions being fuzzy stochastic processes. In this case the notion of the stochastic Itô's integral in the equation is crisp; that is, it has single-valued level sets. The second aim of this paper is to show that there is no extension to more general diffusion terms.
\end{abstract}

\section{Introduction}

Deterministic fuzzy differential equations have been developed due to investigations of dynamic systems where the information on parameters of such systems is incomplete or vague. They play an important role in an increasing number of system models in biology [1], engineering [2], civil engineering [3], bioinformatics and computational biology [4], quantum optics and gravity [5], and hydraulic [6, 7] and modeling of mechanical systems [8]. Many investigations in this area were developed using different approaches for formulations of differential problems in a fuzzy setting (see, e.g., $[1,9-23]$ and references therein). Historically, the earliest approach for deterministic fuzzy differential equations was based on a generalization of the Hukuhara derivative of a set-valued function. This was made by Puri and Ralescu in $[24]$ and used by Kaleva in $[15,16]$. Further extensions were developed next also in [25-27] where the concept of strongly generalized differentiability was introduced. A different approach was proposed by Hüllermeier in [13] where fuzzy differential equations were interpreted as a family of differential inclusions associated with level sets of their fuzzy right hand sides. Such an approach has been also used next among others in $[1,9,12,18,22,28,29]$ (see also the references therein). A further step is a research concerning stochastic fuzzy differential (or integral) equations which generalize both classical stochastic differential equations and deterministic fuzzy differential equations. In this case, the main problem is a concept of a fuzzy stochastic integral which should cover the notion of the classical stochastic Itô integral. A research concerning stochastic fuzzy differential (or integral) equations driven by a Wiener process has been initiated in different forms in [30-33], and it can be applied in modeling of phenomenons where two kinds of uncertainties, that is, randomness and fuzziness, are incorporated simultaneously. For applications in stochastic population models, see, for example, [31, 34]. The aim of this paper is twofold. Firsly, we present a survey of extensions of some of approaches for deterministic fuzzy differential equations to fuzzy stochastic equations driven by semimartingales presented mainly in [35-37] and developed further in [38, 39]. Secondly, we present an analysis of the notion of fuzzy Itô's stochastic integral understood as fuzzy random variable. So, we will start with presentation of some recent results where different 
approaches to the notion of a fuzzy stochastic equation were used. They reflect those known from deterministic case. The first one presented in Section 3 treats a fuzzy equation as an abstract relation in the metric space of fuzzy sets over the space of square integrable random vectors. In the second one we solve an equation via stochastic inclusions approach. In this case, the idea is to solve those inclusions and then apply the theorem of Negoita and Ralescu. In the deterministic case these studies reduce to investigations presented in [9, 13, 15, 28]. In Section 4, we discuss another concept of the notion of fuzzy stochastic differential equations which was developed in [33, 34, 40-44] and which again reduces to classical Kaleva's approach in the deterministic case. Now the idea is to use an embedding of a single-valued stochastic integral driven by a Wiener process into the space of fuzzy sets. Here the advantage is that a solution of fuzzy integral equation appears as a fuzzy-valued stochastic process. On the other hand, the main disadvantage in this case is that the fuzzy-valued stochastic integral is trivial; that is, it has singlevalued level sets. In fact, in this case the integrand must have single-valued level sets as well. Therefore in the context of the study of stochastic fuzzy equation the following problem appears: is it possible to consider fuzzy stochastic differential equations with more general fuzzy-valued diffusion terms and with solutions still being fuzzy-valued stochastic processes? The second aim and the novelty of the paper is the analysis of this problem. This is done in Section 4 where we show that because of unboundedness in general of a fuzzy stochastic Itô's integral such extension may not hold.

\section{Fuzzy Random Variables and Fuzzy Stochastic Integral}

We start with some facts from stochastic analysis needed in the sequel. We recall the notion of a fuzzy stochastic integral with respect to semimartingale integrators studied first in [32] and next used and developed in [35-38]. Let $T>0$ and let $I=[0, T]$ or $R_{+}$. Let $\left(\Omega, \mathbf{F},\left\{\mathbf{F}_{t}\right\}_{t \in I}, P\right)$ be a complete filtered probability space satisfying the usual hypothesis; that is, $\left\{\mathbf{F}_{t}\right\}_{t \in I}$ is an increasing and right continuous family of sub$\sigma$-fields of $\mathbf{F}$ and $\mathbf{F}_{0}$ contains all $P$-null sets. Let $\mathscr{P}$ denote the smallest $\sigma$-field on $I \times \Omega$ with respect to which every leftcontinuous and $\left\{\mathbf{F}_{t}\right\}_{t \in I^{-}}$adapted process is measurable. An $R^{d}$-valued stochastic process $x$ is said to be predictable if $x$ is $\mathscr{P}$-measurable. One has $\mathscr{P} \subset \beta \otimes \mathbf{F}$, where $\beta$ denotes the Borel $\sigma$-field on $I$. Let $L^{2, d}:=L^{2}\left(\Omega, \mathbf{F}, P ; R^{d}\right)$ for $d \geq 1$. By $S^{2}$ we denote the space of all $\left\{\mathbf{F}_{t}\right\}_{t \in I}$-adapted and càdlàg (i.e., right continuous and with finite left-hand limits) processes $\left(x_{t}\right)_{t \in I}$ such that the norm $\|x\|_{S^{2}}:=\left\|\sup _{t \in I}\left|x_{t}\right|\right\|_{L^{2,1}}$ is finite. It is well known that $\left(S^{2},\|\cdot\|_{S^{2}}\right)$ is a Banach space (see, e.g., [45]). We will use the notation $x_{t-}:=\lim _{s>t} x_{t} P$-a.s. Let $Z$ be an $\left\{\mathbf{F}_{t}\right\}_{t \in I^{-}}$-adapted and càdlàg process with values in $R^{1}$. It is said to be a semimartingale if $Z=M+A$, where $M$ is an $\left\{\mathbf{F}_{t}\right\}_{t \in I}$-adapted local martingale and $A$ is an $\left\{\mathbf{F}_{t}\right\}_{t \in I}$-adapted, càdlàg process with finite variation on compact intervals in $I$ (see, e.g., [45] for details). We will assume that $Z_{0-}=Z_{0}=0$. Let us consider the class of $\mathscr{H}^{2}$-semimartingales, that is, the space of $\left\{\mathbf{F}_{t}\right\}_{t \in I}$-adapted semimartingales with a finite $\mathscr{H}^{2}$. norm:

$$
\|Z\|_{\mathscr{H}^{2}}:=\left\|[M, M]_{\sup I}^{1 / 2}\right\|_{L^{2,1}}+\left\|\left(\int_{0}^{\sup I}\left|d A_{t}\right|\right)\right\|_{L^{2,1}}<\infty,
$$

where $[M, M]$ denotes the quadratic variation process for a local martingale $M$, while $|A| .:=\int_{0}^{\cdot}\left|d A_{s}\right|$ represents the total variation of the random measure induced by the paths of the process $A$. Proceeding, similarly as in [32], we will introduce some measure $\mu_{Z}$ on the predictable $\sigma$-field $\mathscr{P}$ associated with a semimartingale $Z$. Since $Z \in \mathscr{H}^{2}$, it follows that $M$ is a square integrable martingale such that $E M_{t}^{2}=E[M, M]_{t}$ for all $t \in I$. By the same reason, the process $A$ has a square integrable total variation on $I$. By $\mu_{M}$ denote the Doléans-Dade measure for the martingale $M$; that is, $\mu_{M}$ is a unique measure on a predictable $\sigma$-field $\mathscr{P}$ such that

$$
\begin{aligned}
& \mu_{M}((s, t] \times A)=E\left(I_{A}\left(M_{t}-M_{s}\right)^{2}\right), \\
& \mu_{M}\left(\{0\} \times A_{0}\right)=0
\end{aligned}
$$

for all $A \in \mathbf{F}_{s}, 0 \leq s<t$, and $A_{0} \in \mathbf{F}_{0}$ (see, e.g., [46]). Then for all $f \in L^{2}\left(I \times \Omega, \mathscr{P}, \mu_{M} ; R^{d}\right)$ the stochastic integral $\int f_{s} d M_{s}$ exists and one has

$$
\begin{aligned}
E\left(\left\|\int_{0}^{t} f_{s} d M_{s}\right\|_{R^{d}}^{2}\right) & =\int_{[0, t] \times \Omega}\|f\|_{R^{d}}^{2} d \mu_{M} \\
& =E\left(\int_{0}^{t}\left\|f_{s}\right\|_{R^{d}}^{2} d[M, M]_{s}\right),
\end{aligned}
$$

for $t \in I$. Let us define a random measure on $I$

$$
\gamma(\omega, d t):=|A(\omega)|_{\sup I}\left|d A_{t}(\omega)\right|
$$

and a measure associated with the process $A$ by the following formula:

$$
\nu_{A}(C):=\int_{\Omega} \int_{0}^{\sup I} I_{C}(\omega, t) \gamma(\omega, d t) P(d \omega)
$$

for every $C \in \mathscr{P}$. Then $v_{A}$ is a finite measure on $\mathscr{P}$. Finally, we define a finite measure $\mu_{Z}$ associated with $Z \in \mathscr{H}^{2}$ by $\mu_{Z}:=\mu_{M}+\nu_{A}$. Let us denote $L_{\mathscr{P}}^{2}\left(\mu_{Z}\right):=L^{2}\left(I \times \Omega, \mathscr{P}, \mu_{Z}\right.$; $\left.R^{d}\right)$. Then by Proposition 1 in [32] for every $f \in L_{\mathscr{P}}^{2}\left(\mu_{Z}\right)$ and $t \in I$ there exists a stochastic integral $\int_{0}^{t} f_{s} d Z_{s}$. In order to introduce a notion of fuzzy stochastic integral we begin with some auxiliary facts. Let $\mathfrak{X}$ be a separable Banach space. By $\mathscr{K}(\mathfrak{X}), \mathscr{K}^{b}(\mathfrak{X})$, or $\mathscr{K}_{c}^{b}(\mathfrak{X})$ we denote the family of all nonempty closed, all nonempty closed and bounded or nonempty closed bonded, and convex subsets of $\mathfrak{X}$, respectively. We will consider the spaces $\mathscr{K}^{b}(\mathfrak{X})$ or $\mathscr{K}_{c}^{b}(\mathfrak{X})$ with a Hausdorff metric $H_{\mathfrak{X}}$ :

$$
H_{\mathfrak{X}}(A, B):=\max \left\{\sup _{a \in A} d_{\mathfrak{X}}(a, B), \sup _{b \in B} d_{\mathfrak{X}}(b, A)\right\},
$$

where $d_{\mathfrak{X}}(a, B):=\inf _{b \in B}\|a-b\|_{\mathfrak{X}}$ and $\|\cdot\|_{\mathfrak{X}}$ is a norm in $\mathfrak{X}$. Then $\left(\mathscr{K}^{b}(\mathfrak{X}), H_{\mathfrak{X}}\right)$ and $\left(\mathscr{K}_{c}^{b}(\mathfrak{X}), H_{\mathfrak{X}}\right)$ are complete metric 
space (cf. [47]). For $A \in \mathscr{K}(\mathfrak{X})$, we set $\||A|\|_{\mathfrak{X}}:=H_{\mathfrak{X}}(A,\{0\})=$ $\sup _{a \in A}\|a\|_{\mathfrak{X}}$. By a fuzzy set $u$ of a Banach space $\mathfrak{X}$ we mean a mapping $u: \mathfrak{X} \rightarrow[0,1]$. The space of all fuzzy sets of $\mathfrak{X}$ will be denoted by the symbol $\mathscr{F}(\mathfrak{X})$. For $\alpha \in(0,1]$ let $[u]^{\alpha}:=\{x \in \mathfrak{X}: u(x) \geq \alpha\}$ and $[u]^{0}:=\operatorname{cl}_{\mathfrak{X}}\{x \in \mathfrak{X}: u(x)>0\}$ where $\mathrm{cl}_{\mathfrak{X}}$ denotes the closure in $\left(\mathfrak{X},\|\cdot\|_{\mathfrak{X}}\right)$. In the sequel we deal with the following fuzzy sets:

$$
\begin{aligned}
& \mathscr{F}^{b}(\mathfrak{X}) \\
& \quad=\left\{u \in \mathscr{F}(\mathfrak{X}):[u]^{\alpha} \in \mathscr{K}^{b}(\mathfrak{X}) \text { for } \alpha \in[0,1]\right\}, \\
& \mathscr{F}_{c}^{b}(\mathfrak{X}) \\
& \quad=\left\{u \in \mathscr{F}(\mathfrak{X}):[u]^{\alpha} \in \mathscr{K}_{c}^{b}(\mathfrak{X}) \text { for } \alpha \in[0,1]\right\} .
\end{aligned}
$$

We will use a metric $D_{\mathfrak{X}}$ in $\mathscr{F}_{c}^{b}(\mathfrak{X})$ described as follows:

$$
D_{X}(u, v):=\sup _{\alpha \in[0,1]} H_{X}\left([u]^{\alpha},[v]^{\alpha}\right)
$$

for $u, v \in \mathscr{F}_{c}^{b}(\mathfrak{X})$.

One can show (cf. [48]) that $\left(\mathscr{F}_{c}^{b}(\mathfrak{X}), D_{\mathfrak{X}}\right)$ is a complete metric space. Other metrics used in the set $\mathscr{F}_{c}^{b}(\mathfrak{X})\left(\operatorname{apart} D_{\mathfrak{X}}\right)$ are

$$
D_{p}^{\mathfrak{X}}(u, v):=\left(\int_{0}^{1} H_{\mathfrak{X}}^{p}\left([u]^{\alpha},[v]^{\alpha}\right) d \alpha\right)^{1 / p}, \quad p \geq 1,
$$

and the Skorokhod metric

$$
D_{S}^{\mathfrak{X}}(u, v):=\inf _{\lambda \in \Lambda} \max \{a, b\},
$$

where $a=\sup _{t \in[0,1]}|\lambda(t)-t|, b=\sup _{t \in[0,1]} d_{H}\left(x_{u}(t), x_{v}(\lambda(t))\right)$, and $\Lambda$ is the set of strictly increasing continuous functions $\lambda:[0,1] \rightarrow[0,1]$ with $\lambda(0)=0$ and $\lambda(1)=1$. Functions $x_{u}, x_{v}:[0,1] \rightarrow \mathscr{K}_{c}^{b}(\mathfrak{X})$ are the càdlàg representations for the fuzzy sets $u, v \in \mathscr{F}_{c}^{b}(\mathfrak{X})$; see Colubi et al. [49] for details. The space $\left(\mathscr{F}_{c}^{b}(\mathfrak{X}), D_{p}^{\mathfrak{X}}\right)$ is separable and noncomplete but $\left(\mathscr{F}_{c}^{b}(\mathfrak{X}), D_{S}^{\mathfrak{X}}\right)$ is a Polish metric space. By $\mathscr{B}_{\rho}$, we define a borel $\sigma$-field in a metric space $\left(\mathscr{F}_{c}^{b}(\mathfrak{X}), \rho\right)$ where $\rho$ is one of metrics described above. For $u, v \in \mathscr{F}_{c}^{b}(\mathfrak{X})$ the addition $u \oplus v$ is defined by Zadeh's extension principle (see [11]). But, due to Lemma 3.4 in [50], it can be also defined levelwise; that is,

$$
[u \oplus v]^{\alpha}=[u]^{\alpha}+[v]^{\alpha} \quad \text { for each } \alpha \in[0,1] .
$$

In what follows, we will use the following version of the theorem of Negoita and Ralescu.

Theorem 1 (see [51]). Let $Y \subset \mathfrak{X}$ be a nonempty set and let $\left\{Y_{\alpha}: \alpha \in[0,1]\right\}$ be a family of subsets of $Y$ such that
(a) $Y_{0}=Y$,
(b) $Y_{0} \supset Y_{\alpha} \supset Y_{\beta}$ for $0 \leq \alpha \leq \beta$,
(c) if $\alpha_{n} \nearrow \alpha$ then $Y_{\alpha}=\bigcap_{n=1}^{\infty} Y_{\alpha_{n}}$.

Then there exists $u \in \mathscr{F}(\mathfrak{X})$ such that $[u]^{\alpha}=Y_{\alpha}$ for every $\alpha \in(0,1]$, and $[u]^{0} \subset Y_{0}$. Moreover

$$
u(x)= \begin{cases}\sup \left\{\alpha: x \in Y_{\alpha}\right\}, & \text { if } x \in Y, \\ 0, & \text { if } x \notin Y .\end{cases}
$$

On the other hand for $u \in \mathscr{F}(\mathfrak{X})$ the family of sets $Y_{0}:=[u]^{0}$ and $Y_{\alpha}:=[u]^{\alpha}, \alpha \in(0,1]$, satisfies conditions $(b)$ and $(c)$.

Let $f: \Omega \rightarrow \mathscr{F}_{c}^{b}(\mathfrak{X})$ be such that $[f(\cdot)]^{\alpha}: \Omega \rightarrow \mathscr{K}_{c}^{b}(\mathfrak{X})$ is an F-measurable set-valued mapping (in the sense of setvalued analysis, see, e.g., [47]) for every $\alpha \in[0,1]$. Then $f$ is called a fuzzy random variable (in the sense of Puri and Ralescu). A fuzzy-valued mapping $f: I \times \Omega \rightarrow \mathscr{F}_{c}^{b}\left(R^{d}\right)$ is said to be a predictable fuzzy-valued stochastic process, if the set-valued function $[f]^{\alpha}: I \times \Omega \rightarrow \mathscr{K}_{c}^{b}\left(R^{d}\right),[f]^{\alpha}(t, \omega):=$ $[f(t, \omega)]^{\alpha}$ is $\mathscr{P}$-measurable. We call $f$ to be $L_{\mathscr{P}}^{2}\left(\mu_{Z}\right)$-integrally bounded if $\left\|\left|[f]^{0}\right|\right\| \in L^{2}\left(I \times \Omega, \mathscr{P}, \mu_{Z}, R_{+}\right)$. Taking such a predictable fuzzy-valued stochastic process $f$, let us consider (for every fixed $\alpha \in[0,1]$ ) the following set:

$$
S_{\mathscr{P}}^{2}\left([f]^{\alpha}, \mu_{Z}\right):=\left\{u \in L_{\mathscr{P}}^{2}\left(\mu_{Z}\right): u \in[f]^{\alpha} \mu_{Z} \text { a.e. }\right\} .
$$

Then by Kuratowski and Ryll-Nardzewski Selection Theorem (see, e.g., [52]), it follows that $S_{\mathscr{P}}^{2}\left([f]^{\alpha}, \mu_{Z}\right) \neq \emptyset$ for $\alpha \in[0,1]$. Hence for every $u \in S_{\mathscr{P}}^{2}\left([f]^{\alpha}, \mu_{Z}\right)$ the stochastic integral $\int_{0}^{t} u_{s} d Z_{s}$ exists for $t \in I$. Consequently, for every $t \in I$ and $\alpha \in[0,1]$ one can consider [32] the set

$$
\int_{0}^{t}[f]_{s}^{\alpha} d Z_{s}:=\left\{\int_{0}^{t} u_{s} d Z_{s}: u \in S_{\mathscr{P}}^{2}\left([f]^{\alpha}, \mu_{Z}\right)\right\} .
$$

The set $\int_{0}^{t}[f]_{s}^{\alpha} d Z_{s}$ is called set-valued stochastic integral of the set-valued mapping $[f]^{\alpha}: I \times \Omega \rightarrow \mathscr{K}_{c}^{b}\left(R^{d}\right)$ with respect to semimartingale $Z$.

Below we collect the main properties of the sets $S_{\mathscr{P}}^{2}\left([f]^{\alpha}\right.$, $\left.\mu_{Z}\right)$ and $\int_{0}^{t}[f]_{s}^{\alpha} d Z_{s}$ for $t \in I$ and $\alpha \in[0,1]$.

Proposition 2 (see [32]). Let $f: I \times \Omega \rightarrow \mathscr{F}_{c}^{b}\left(R^{d}\right)$ be a predictable and $L_{\mathscr{P}}^{2}\left(\mu_{Z}\right)$-integrally bounded fuzzy-valued mapping. Then

(a) $S_{\mathscr{P}}^{2}\left([f]^{\alpha}, \mu_{Z}\right)$ is a closed, convex, bounded, weakly compact, and decomposable subset of $L_{\mathscr{P}}^{2}\left(\mu_{Z}\right)$ for every $\alpha \in[0,1]$,

(b) The set $\int_{0}^{t}[f]_{s}^{\alpha} d Z_{s}$ is a bounded closed, weakly compact, and convex subset of $L^{2, d}$ for every $t \in I$ and $\alpha \in[0,1]$.

Theorem 3 (see [32]). Let $f: I \times \Omega \rightarrow \mathscr{F}_{c}^{b}\left(R^{d}\right)$ be a predictable and $L_{\mathscr{P}}^{2}\left(\mu_{Z}\right)$-integrally bounded fuzzy-valued mapping. Then one has the following:

(a)

$$
\int_{0}^{t}[f]_{s}^{\alpha_{1}} d Z_{s} \supset \int_{0}^{t}[f]_{s}^{\alpha_{2}} d Z_{s}
$$

for every $t \in I$ and $\alpha_{1} \leq \alpha_{2}$. 
(b)

$$
\int_{0}^{t}[f]_{s}^{\alpha} d Z_{s}=\bigcap_{n \geq 1} \int_{0}^{t}[f]_{s}^{\alpha_{n}} d Z_{s}
$$

for every $t \in I$ and $\alpha_{n} \nearrow \alpha$.

Then by Theorem 1, Proposition 2, and Theorem 3, for every fixed $t \in I$, there exists a fuzzy set (say) $X(f, Z)_{t} \in$ $\mathscr{F}_{c}^{b}\left(L^{2, d}\right)$ such that $\left[X(f, Z)_{t}\right]^{\alpha}=\int_{0}^{t}[f]_{s}^{\alpha} d Z_{s}$ for every $t \in I$ and every $\alpha \in[0,1]$. This leads to the following definition (see [32]).

Definition 4. By a fuzzy stochastic integral (over the interval $[0, t])$ of the predictable and $L_{\mathscr{P}}^{2}\left(\mu_{Z}\right)$-integrally bounded fuzzy stochastic process $f$ with respect to the semimartingale $Z$, one means a fuzzy set $X(f, Z)_{t}$ described above. One denotes it by $X(f, Z)_{t}:=(\mathscr{F}) \int_{0}^{t} f(s) d Z_{s}, t \in I$.

Using similar methods as in [36] and general properties of stochastic integrals driven by càdlàg semimartingales, one can show the following properties.

Proposition 5. Let $f, f_{1}, f_{2}: I \times \Omega \rightarrow \mathscr{F}_{c}^{b}\left(R^{d}\right)$ be predictable and $L_{\mathscr{P}}^{2}\left(\mu_{Z}\right)$-integrally bounded fuzzy-valued functions. Then for all $\tau, a, t, \tau \leq a \leq t$ it holds:

(a)

$$
\begin{aligned}
(\mathscr{F}) \int_{\tau}^{t} f(s) d Z_{s}= & (\mathscr{F}) \int_{\tau}^{a} f(s) d Z_{s} \\
& \oplus(\mathscr{F}) \int_{a}^{t} f(s) d Z_{s}
\end{aligned}
$$

(b)

$$
\begin{aligned}
& D_{L^{2, d}}^{2}\left((\mathscr{F}) \int_{\tau}^{t} f_{1}(s) d Z_{s},(\mathscr{F}) \int_{\tau}^{t} f_{2}(s) d Z_{s}\right) \\
& \quad \leq 2 \int_{(\tau, t] \times \Omega} D_{R^{d}}^{2}\left(f_{1}, f_{2}\right) d \mu_{Z} .
\end{aligned}
$$

(c) The mapping

$$
[\tau, \infty) \ni t \longmapsto(\mathscr{F}) \int_{\tau}^{t} f(s) d Z_{s} \in \mathscr{F}_{c}^{b}\left(L^{2, d}\right)
$$

is right continuous with finite left-hand limits with respect to the metric $D_{L^{2, d}}$.

Remark 6. In [38] a slight extension of the notion of fuzzy stochastic integral with respect to the semimartingale $Z=$ $A+M$ was proposed. Namely, it was defined as the sum:

$$
(\mathscr{F}) \int_{0}^{t} f(s) d A_{s} \oplus(\mathscr{F}) \int_{0}^{t} f(s) d M_{s} .
$$

Although, in general, the fuzzy sets $(\mathscr{F}) \int_{0}^{t} f(s) d Z_{s}$ and $(\mathscr{F}) \int_{0}^{t} f(s) d A_{s} \oplus(\mathscr{F}) \int_{0}^{t} f(s) d M_{s}$ are different, however the main properties of the sum $(\mathscr{F}) \int_{0}^{t} f(s) d A_{s} \oplus(\mathscr{F}) \int_{0}^{t} f(s) d M_{s}$ follow easily from the same ideas as in the case of the fuzzy set $(\mathscr{F}) \int_{0}^{t} f(s) d Z_{s}$ for $Z=A$ and $Z=M$, respectively (see [35]).

Remark 7. In Section 4, we will study the notion of fuzzy stochastic integral driven by the Wiener process and which is understood as a fuzzy-valued random variable. We will show there that such understood fuzzy-valued stochastic integrals may have unbounded in $L^{2}$ level sets.

\section{Fuzzy Stochastic Differential Equation Driven by a Semimartingale}

Below we establish recent result for two different approaches for fuzzy stochastic differential equations.

3.1. A Direct Approach. For further considerations, we assume that the $\sigma$-field $\mathbf{F}$ is separable with respect to the probability measure $P$ and $Z$ is a continuous semimartingale. A direct approach is based on the framework of the metric space $\left(\mathscr{F}_{c}^{b}\left(L^{2, d}\right), D_{L^{2, d}}\right)$ (see [36]). Therefore, in this case by a stochastic fuzzy differential equation (written in its integral form), we mean the following relation in the space $\left(\mathscr{F}_{c}^{b}\left(L^{2, d}\right), D_{L^{2, d}}\right)$ :

$$
X(t)=X_{0} \oplus(\mathscr{F}) \int_{0}^{t} f(s, X(s)) d Z_{s}, \quad t \in I,
$$

where $f: I \times \Omega \times \mathscr{F}_{c}^{b}\left(L^{2, d}\right) \rightarrow \mathscr{F}_{c}^{b}\left(R^{d}\right)$ is a given fuzzy-valued mapping and $X_{0} \in \mathscr{F}_{c}^{b}\left(L_{0}^{2, d}\right)$ with $L_{0}^{2, d}:=L^{2}\left(\Omega, \mathbf{F}_{0}, P ; R^{d}\right)$.

Definition 8. By a solution to (21), one means a $D_{L^{2, d}-}$ continuous mapping $X: I \rightarrow \mathscr{F}_{c}^{b}\left(L^{2, d}\right)$ such that $(21)$ is satisfied. The solution $X$ of $(21)$ is unique, if $X(t)=Y(t)$ for all $t \in I$ where $Y: I \rightarrow \mathscr{F}_{c}^{b}\left(L^{2, d}\right)$ is any other solution of $(21)$.

Let $\theta$ and $\Theta$ denote zero elements in $R^{d}$ and $L^{2, d}$, respectively. By $\widehat{\theta}$ and $\widehat{\Theta}$, we denote their fuzzy counterparts; that is, $\widehat{\theta} \in \mathscr{F}_{c}^{b}\left(R^{d}\right)$ such that $[\hat{\theta}]^{\alpha}=\{\theta\}$ for every $\alpha \in[0,1]$, and similarly $\widehat{\Theta} \in \mathscr{F}_{c}^{b}\left(L^{2, d}\right)$ with $[\widehat{\Theta}]^{\alpha}=\{\Theta\}$ for every $\alpha \in[0,1]$. We assume that $f: I \times \Omega \times \mathscr{F}_{c}^{b}\left(L^{2, d}\right) \rightarrow \mathscr{F}_{c}^{b}\left(R^{d}\right)$ satisfies the following conditions:

(f1) $f$ is $\mathscr{P} \otimes \mathscr{B}_{D_{S}^{L^{2, d}}} \mid \mathscr{B}_{D_{S}^{\mathbb{R}^{d}}}$-measurable.

(f2) There exists a constant $K>0$ such that $P$-a.e. holds:

$$
D_{R^{d}}(f(t, \omega, u), f(t, \omega, v)) \leq K D_{L^{2, d}}(u, v)
$$

for all $t \in I$ and every $u, v \in \mathscr{F}_{c}^{b}\left(L^{2, d}\right)$.

(f3) There exists a constant $C>0$ such that $P$-a.e. holds:

$$
D_{R^{d}}(f(t, \omega, u), \widehat{\theta}) \leq C\left(1+D_{L^{2, d}}(u, \widehat{\Theta})\right)
$$

for all $t \in I$ and every $u \in \mathscr{F}_{c}^{b}\left(L^{2, d}\right)$. 
Then using fixed point argument and continuation procedure, one can show the following (see [36]).

Theorem 9. Let $X_{0} \in \mathscr{F}_{c}^{b}\left(L_{0}^{2, d}\right)$ and let $f: I \times \Omega \times \mathscr{F}_{c}^{b}\left(L^{2, d}\right) \rightarrow$ $\mathscr{F}_{c}^{b}\left(R^{d}\right)$ satisfy $(f 1)-(f 3)$. Then there exists a unique solution to (21) in the sense of Definition 8.

Now we consider (21) and equation with another initial value $Y_{0} \in \mathscr{F}_{c}^{b}\left(L_{0}^{2, d}\right)$; that is

$$
Y(t)=Y_{0} \oplus(\mathscr{F}) \int_{0}^{t} f(s, Y(s)) d Z_{s}, \quad t \in I .
$$

Let $X, Y$ denote the solutions to (21) and (24), respectively. Then the following result holds (see [36]).

Theorem 10. Let $X_{0}, Y_{0} \in \mathscr{F}_{c}^{b}\left(L_{0}^{2, d}\right)$ and let $f: I \times \Omega \times$ $\mathscr{F}_{c}^{b}\left(L^{2, d}\right) \rightarrow \mathscr{F}_{c}^{b}\left(R^{d}\right)$ satisfy conditions $(f 1)-(f 3)$. Then

$$
\begin{array}{r}
D_{L^{2, d}}(X(t), Y(t)) \leq \sqrt{2} e^{2 K^{2} \mu_{Z}([0, t] \times \Omega)} D_{L^{2, d}}\left(X_{0}, Y_{0}\right) \\
\text { for every } t \in I .
\end{array}
$$

Corollary 11. Under assumptions of Theorem 10, we have

$$
\sup _{t \in I} D_{L^{2, d}}(X(t), Y(t)) \leq \sqrt{2} e^{2 K^{2} \mu_{Z}(I \times \Omega)} D_{L^{2, d}}\left(X_{0}, Y_{0}\right) .
$$

For the stability of solution with respect to the right-hand side, we consider the following sequence of equations (for $n=$ $1,2, \ldots)$ :

$$
X_{n}(t)=X_{0} \oplus(\mathscr{F}) \int_{0}^{t} f_{n}\left(s, X_{n}(s)\right) d Z_{s}, \quad t \in I .
$$

By $X, X_{n}$ we denote the corresponding solutions to (21) and (27). Then we have [36].

Theorem 12. Let $X_{0} \in \mathscr{F}_{c}^{b}\left(L_{0}^{2, d}\right)$. Suppose that $f, f_{n}: I \times \Omega \times$ $\mathscr{F}_{c}^{b}\left(L^{2, d}\right) \rightarrow \mathscr{F}_{c}^{b}\left(R^{d}\right)$ satisfy conditions $(f 1)-(f 3)$ with the same Lipschitz constant $K$. If for every $t \in I$ and every $u \in \mathscr{F}_{c}^{b}\left(L^{2, d}\right)$

$$
\int_{[0, t] \times \Omega} D_{R^{d}}^{2}\left(f_{n}(s, u), f(s, u)\right) d \mu_{Z} \longrightarrow 0,
$$

$$
\text { as } n \longrightarrow \infty \text {, }
$$

then for every $t \in I$

$$
D_{L^{2, d}}\left(X_{n}(t), X(t)\right) \longrightarrow 0, \quad \text { as } n \longrightarrow \infty .
$$

Corollary 13. Under assumptions of Theorem 12 one has

$$
\sup _{t \in I} D_{L^{2, d}}\left(X_{n}(t), X(t)\right) \longrightarrow 0, \quad \text { as } n \longrightarrow \infty,
$$

provided that for every $u \in \mathscr{F}_{c}^{b}\left(L^{2, d}\right)$

$$
\int_{I \times \Omega} D_{R^{d}}^{2}\left(f_{n}(s, u), f(s, u)\right) d \mu_{Z} \longrightarrow 0,
$$

Remark 14. Setting $Z$ as $Z(t)=t$ for $t \in I, f: I \times \mathscr{F}_{c}^{b}\left(R^{d}\right) \rightarrow$ $\mathscr{F}_{c}^{b}\left(R^{d}\right)$, and $X_{0} \in \mathscr{F}_{c}^{b}\left(R^{d}\right)$ in (21), we get the fuzzy initial value problem:

$$
\begin{aligned}
X^{\prime}(t) & =f(t, X(t)), \\
X(0) & =X_{0},
\end{aligned}
$$

where ' denotes the fuzzy Hukuhara derivative (cf. [11, 15, $16,20]$ and references therein). Assumptions (f1)-(f3) in this case reduce to the classical measurability, Lipschitz, and growth conditions. Thus by Theorem 9, (32) admits a unique solution. Also in this case, Theorems 10 and 12 and Corollaries 11 and 13 hold.

Remark 15. Using similar methods as in [35], one can prove existence and uniqueness results to fuzzy stochastic integral equation (with respect to continuous $\mathscr{H}^{2}$-semimartingale $Z=A+M)$

$$
\begin{aligned}
X(t)= & X_{0} \oplus(\mathscr{F}) \int_{0}^{t} f(s, X(s)) d A_{s} \\
& \oplus(\mathscr{F}) \int_{0}^{t} f(s, X(s)) d M_{s}, \quad t \in I,
\end{aligned}
$$

or to its $m$-component version. Such results were established in [38]. Further considerations for fuzzy stochastic equations in the metric space $\left(\mathscr{F}_{c}^{b}\left(L^{2, d}\right), D_{L^{2, d}}\right)$ setup and under weaker assumptions than Lipschitz type can be found in [39].

3.2. A Stochastic Inclusion Approach. In this part, we present a stochastic counterpart of the approach for the notion of deterministic fuzzy differential equations proposed among others in $[1,9,12,13,18-20,22,29]$ where the fuzzy differential equation has been meant as a system of differential inclusions. As previously, let $Z$ be a given $\mathscr{H}^{2}$-semimartingale on a filtered probability space $\left(\Omega, \mathbf{F},\left\{\mathbf{F}_{t}\right\}_{t \in I}, P\right)$. Let us recall that by $S^{2}$ we denote the space of all $\left\{\mathbf{F}_{t}\right\}_{t \in I^{-}}$-adapted and càdlàg processes $\left(x_{t}\right)_{t \in I}$ such that the norm $\|x\|_{S^{2}}:=\left\|\sup _{t \in I}\left|x_{t}\right|\right\|_{L^{2,1}}$ is finite. By $\langle\cdot\rangle: L_{0}^{2, d} \rightarrow \mathscr{F}_{c}^{b}\left(L_{0}^{2, d}\right)$ we denote an embedding of $L_{0}^{2, d}$ into $\mathscr{F}_{c}^{b}\left(L_{0}^{2, d}\right)$; that is, for $a \in L_{0}^{2, d}$, we have $\langle a\rangle(z)=$ $\square_{\{a\}}(z)$ for $z \in L_{0}^{2, d}$. We consider a fuzzy-valued function $f: I \times \Omega \times R^{d} \rightarrow \mathscr{F}_{c}^{b}\left(R^{d}\right)$ and $\xi \in L_{0}^{2, d}$. We consider now the formal relation (called here as a fuzzy stochastic differential equation)

$$
\begin{aligned}
d X_{t} & =f\left(t, X_{t-}\right) d Z_{t}, \quad t \in I, \\
X_{0} & =\langle\xi\rangle .
\end{aligned}
$$

Following [37], it is interpreted as a family of stochastic integral inclusions

$$
\begin{aligned}
x_{t}-x_{s} & \in \int_{s}^{t}\left[f\left(\tau, x_{\tau-}\right)\right]^{\alpha} d Z_{\tau}, \quad 0 \leq s<t \leq T, \\
x_{0} & =\xi
\end{aligned}
$$

for $\alpha \in[0,1]$. It can be shown that, under appropriate assumptions, solution sets of (35) are bounded and closed in a 
Banach space $S^{2}$ and they generate a fuzzy set in $S^{2}$. Therefore, for a fixed $\alpha \in[0,1]$, we define first the notion of a solution to (35). Namely, by a strong solution to stochastic inclusion (35), we mean a càdlàg and an $\left\{\mathbf{F}_{t}\right\}_{t \in I}$-adapted stochastic process $x=\left(x_{t}\right)_{t \in I}, x \in S^{2}$, such that

$$
x_{t}=\xi+\int_{0}^{t} a_{s} d Z_{s}
$$

$$
\text { for } a \in S_{\mathscr{P}}^{2}\left(\left[f \circ x_{-}\right]^{\alpha}, \mu_{Z}\right), t \in I \text {, }
$$

where $\left[f \circ x_{-}\right]^{\alpha}(t, \omega)=\left[f\left(t, \omega, x_{t-}(\omega)\right]^{\alpha}\right.$. Let $\Gamma(f, \xi, \alpha)$ denote the set of all strong solutions to (35). Then $\Gamma(f, \xi, \alpha) \subset S^{2}$. Suppose $\Gamma(f, \xi, \alpha) \neq \emptyset$. Thus we have the following definition of the fuzzy solution to (34) [37].

Definition 16. By the fuzzy solution to (34), one means a fuzzy set $X(f, \xi) \in \mathscr{F}^{b}\left(S^{2}\right)$ such that $[X(f, \xi)]^{\alpha}=\Gamma(f, \xi, \alpha)$ for $\alpha \epsilon$ $[0,1]$.

To proceed further, we assume the following conditions:

(h1) $Z$ is a given $\left\{\mathbf{F}_{t}\right\}_{t \in I^{-}}$-adapted, $\mathscr{H}^{2}$-semimartingale with a decomposition $Z=M+A$, where $A$ is an $\left\{\mathbf{F}_{t}\right\}_{t \in I^{-}}$ adapted increasing predictable process.

(h2) $\mu_{Z}$ is absolutely continuous with respect to the product measure $\lambda \otimes P$ on the $\sigma$-field $\mathscr{P}$.

(h3) The function $f: I \times \Omega \times R^{d} \rightarrow \mathscr{F}_{c}^{b}\left(R^{d}\right)$ is $\mathscr{P}_{\otimes}$ $\beta\left(R^{d}\right)$-measurable, while $f(\cdot, \cdot, x)$ is $L_{\mathscr{P}}^{2}\left(\mu_{Z}\right)$-integrally bounded for every $x \in R^{d}$.

(h4) There exists a constant $K>0$ such that $D_{R^{d}}(f(t$, $\omega, x), f(t, \omega, y)) \leq K\|x-y\|_{R^{d}}$ for every $x, y \in R^{d}$ and every $(t, \omega) \in I \times \Omega$.

(h5) There exists a constant $C>0$ such that $D_{R^{d}}^{2}(f(t$, $\omega, x), \widehat{\theta}) \leq C\left(1+\|x\|^{2}\right)$ for every $x \in R^{d}$ and every $(t, \omega) \in I \times \Omega$.

Then we have the following result (see [37]).

Theorem 17. If conditions (h1)-(h5) hold, then there exists a fuzzy solution in the sense of Definition 16 to the fuzzy stochastic differential equation (34).

Remark 18. Taking $\xi \in R^{d}$ and $Z_{t}=t$ in (34) for $t \in I$, condition (h1) is satisfied. If $f: I \times \mathbb{R}^{d} \rightarrow \mathscr{F}_{c}^{b}\left(\mathbb{R}^{d}\right)$, then (34) reduces to the deterministic fuzzy differential equation (cf. $[9,13])$ :

$$
\begin{aligned}
d x(t) & =f(t, x(t)) d t \\
x(0) & =\langle\xi\rangle
\end{aligned}
$$

interpreted as the family of integral inclusions:

$$
\begin{gathered}
x(t)-x(s) \in \int_{s}^{t}[f(\tau, x(\tau))]^{\alpha} d \tau, \\
x(0)=\xi
\end{gathered}
$$

for $\alpha \in[0,1]$ and $0 \leq s<t \leq T$. In this case, the space $S^{2}$ coincides with the space of continuous functions $C\left(I, R^{d}\right)$ and we have

$$
\begin{aligned}
& \Gamma(f, \xi, \alpha):=\left\{x \in C\left(I, R^{d}\right): x(t)-x(s)\right. \\
& \left.\quad \in \int_{s}^{t}[f(\tau, x(\tau))]^{\alpha} d \tau, 0 \leq s \leq t \leq T, x(0)=\xi\right\} .
\end{aligned}
$$

Then under conditions (h2), (h3), and (h4) due to Theorem 17 , there exists a fuzzy set $X(f, \xi) \in \mathscr{F}^{b}\left(C\left(I, R^{d}\right)\right.$ being a fuzzy solution to (37) and such that $[X(f, \xi)]^{\alpha}=\Gamma(f, \xi, \alpha)$ for every $\alpha \in[0,1]$. In fact, in this case, the set $\Gamma(f, \xi, \alpha)$ is nonempty and compact in $C\left(I, R^{d}\right)$ for every $\alpha \in[0,1]$. Consequently, the fuzzy solution $X(f, \xi)$ has compact level sets.

Remark 19. In [32] similar approach was used to fuzzy stochastic differential equations driven by the Wiener process. The idea implemented in this case was based on martingale problem approach for the existence of weak solutions to the system of associated stochastic integral inclusions. Thus the existence of fuzzy solutions could be achieved under weaker conditions than Lipschitz continuity imposed on the right-hand side of a fuzzy stochastic differential equation. But also in this case its deterministic counterpart reduces to the one described in Remark 18.

\section{Fuzzy-Valued Stochastic Equations with Fuzzy Stochastic Solutions}

The approaches presented above, that is, direct and based on stochastic inclusions, are different. It is easy to note that they lead to the different notions of fuzzy-valued solutions. In the first one the fuzzy solution is meant as a continuous fuzzyvalued mapping $X: I \rightarrow \mathscr{F}_{c}^{b}\left(L^{2, d}\right)$ satisfying (21), while in the second approach by a solution to (34) we mean an element in $\mathscr{F}^{b}\left(S^{2}\right)$ described in Definition 16. In a series of papers [33, $34,40-44]$ another concept of the notion of fuzzy stochastic integral equation was proposed. Generally speaking, it is understood as the following relation:

$$
\begin{aligned}
x(t)= & x_{0} \oplus(\mathscr{F}) \int_{0}^{t} f(s, x(s)) d s \\
& \oplus\left\langle\int_{0}^{t} g(s, x(s)) d W(s)\right\rangle P \text {-a.e., } t \in I,
\end{aligned}
$$

where $f: I \times \Omega \times \mathscr{F}_{c}^{b}\left(R^{d}\right) \rightarrow \mathscr{F}_{c}^{b}\left(R^{d}\right), g: I \times \Omega \times$ $\mathscr{F}_{c}^{b}\left(R^{d}\right) \rightarrow R^{d}$, and $x_{0}: \Omega \rightarrow \mathscr{F}_{c}^{b}\left(R^{d}\right)$ are appropriately measurable mappings while $W$ is the standard real Wiener process. Similarly as earlier by $\langle\cdot\rangle$ we denote an embedding $\langle\cdot\rangle: R^{d} \rightarrow \mathscr{F}_{c}^{b}\left(R^{d}\right)$ of $R^{d}$ into $\mathscr{F}_{c}^{b}\left(R^{d}\right)$; that is, for $a \in R^{d}$ we have $\langle a\rangle(z)=\mathbb{\square}_{\{a\}}(z)$ for $z \in R^{d}$. Hence $\left\langle\int_{0}^{t} g(s, x(s)) d W(s)\right\rangle$ is fuzzy-valued random variable such that

$$
\left[\left\langle\int_{0}^{t} g(s, x(s)) d W(s)\right\rangle\right]^{\alpha}
$$




$$
\begin{aligned}
& =\int_{0}^{t}[g(s, x(s))]^{\alpha} d W(s) \\
& =\left\{\int_{0}^{t} g(s, x(s)) d W(s)\right\} P \text {-a.e., } \quad t \in I
\end{aligned}
$$

and $\alpha \in[0,1]$. It means that diffusion term above is singlevalued and therefore in fact no fuzziness is here incorporated. The symbol $(\mathscr{F}) \int_{0}^{t} f(s, x(s)) d s$ in (40) denotes a fuzzy-valued Lebesgue stochastic integral such that $\left[(\mathscr{F}) \int_{0}^{t} f(s, x(s)) d s\right]^{\alpha}=(\mathscr{A}) \int_{0}^{t}[f(s, x(s))]^{\alpha} d s$ where the last integral is a set-valued Aumann integral (of set-valued stochastic process $\left.[f \circ x]^{\alpha}: I \times \Omega \rightarrow \mathscr{K}_{c}^{b}\left(R^{d}\right)\right)$ understood as random set for every $t \in I$. Then (see, e.g., [33, 40]) under the classical Lipschitz type assumption, there exists a unique continuous stochastic fuzzy-valued process $x$ being a solution to (40). Unfortunately, in the series of papers mentioned above, only such diffusion case was studied. Therefore one can ask if it is possible to consider fuzzy stochastic differential equations with more general fuzzy-valued diffusion terms and with solutions being fuzzy-valued stochastic processes. Unfortunately, avoiding such trivial diffusion cases the answer is negative in general. As it is shown below this problem is strictly connected with the lack of integrally boundedness in general of set-valued stochastic integrals treated as set-valued random variables even for integrally bounded or bounded integrands. Suppose that for a fuzzy stochastic process $h$ we can define a fuzzy-valued Itô stochastic integral which is assumed to be a fuzzy-valued random variable:

$$
(\mathscr{F}) \int_{0}^{T} h(s) d W(s): \Omega \longrightarrow \mathscr{F}_{c}^{b}\left(R^{d}\right) .
$$

Then for every $\alpha \in[0,1]$ we have a set-valued random mapping

$$
\left[(\mathscr{F}) \int_{0}^{T} h(s) d W(s)\right]^{\alpha}: \Omega \longrightarrow \mathscr{F}_{c}^{b}\left(R^{d}\right)
$$

such that for every $\omega \in \Omega$ the family $\left\{\left[(\mathscr{F}) \int_{0}^{T} h(s) d W(s)\right]^{\alpha}(\omega)\right.$ : $\alpha \in[0,1]\}$ (of convex and compact subsets of $R^{d}$ ) satisfies properties (a), (b), and (c) of Theorem 1, where

$$
\begin{aligned}
& {\left[(\mathscr{F}) \int_{0}^{T} h(s) d W(s)\right]^{\alpha}(\omega)} \\
& \quad:=\left[\left((\mathscr{F}) \int_{0}^{T} h(s) d W(s)\right)(\omega)\right]^{\alpha} \quad \text { for } \omega \in \Omega .
\end{aligned}
$$

In order to have an extension (beyond the crisp case) of a fuzzy stochastic integral as a fuzzy random variable, it should coincide in a crisp case with ordinary (single-valued) Itô's stochastic integral. Thus in the case when $h$ is such that $[h(t, \omega)]^{\alpha}=\{a(t, \omega)\}, \alpha \in[0,1]$, for some $R^{d}$-valued predictable (or nonanticipating in this case) and square integrable stochastic process $a$, it follows that

$$
\begin{aligned}
& {\left[(\mathscr{F}) \int_{0}^{T} h(s) d W(s)\right]^{\alpha}=\left[\left\langle\int_{0}^{T} a(s) d W(s)\right\rangle\right]^{\alpha}} \\
& \quad=\left\{\int_{0}^{T} a(s) d W(s)\right\} P \text {-a.e. }
\end{aligned}
$$

for every $\alpha \in[0,1]$. Thus in general, we should have

$$
\mathcal{S}_{\mathbf{F}_{T}}^{2}\left(\left[(\mathscr{F}) \int_{0}^{T} h d W\right]^{\alpha}, P\right) \supseteq \int_{0}^{T}[h(s)]^{\alpha} d W(s)
$$

for every $\alpha \in[0,1]$, where $\mathcal{S}_{\mathbf{F}_{T}}^{2}\left(\left[(\mathscr{F}) \int_{0}^{T} h d W\right]^{\alpha}, P\right)$ denotes the set of $\mathbf{F}_{T}$-measurable and $L^{2}$-selections of set-valued random variable $\left[(\mathscr{F}) \int_{0}^{T} h d W\right]^{\alpha}$. Note that in this case the set $\int_{0}^{T}[h(s)]^{\alpha} d W(s)$ is defined as in Section 2 and it is a setvalued stochastic integral with respect to a semimartingale which is now a Wiener process. Assume now that $h$ is such that the sets $[h(t, \omega)]^{\alpha}$ are not singletons for all $\alpha \in[0,1]$ and $(t, \omega) \in I \times \Omega$. Then it follows that the sets $\int_{0}^{T}[h(s)]^{\alpha} d W(s)$ need not be singletons as well. Hence they need not be decomposable subsets of the space $L_{T}^{2, d}:=L^{2}\left(\Omega, \mathbf{F}_{T}, P ; R^{d}\right)$ (see $[32,53]$ for details) while the set $\delta_{\mathbf{F}_{T}}^{2}\left(\left[(\mathscr{F}) \int_{0}^{T} h d W\right]^{\alpha}, P\right)$ is closed and decomposable. Hence, we get

$$
\begin{aligned}
& \mathcal{S}_{\mathbf{F}_{T}}^{2}\left(\left[(\mathscr{F}) \int_{0}^{T} h d W\right]^{\alpha}, P\right) \\
& \quad \supseteq \mathrm{cl}_{L_{T}^{2, d}} \operatorname{dec}\left(\int_{0}^{T}[h(s)]^{\alpha} d W(s)\right)
\end{aligned}
$$

for all $\alpha \in[0,1]$, where $\mathrm{cl}_{L_{T}^{2, d}} \operatorname{dec}(A)$ denotes a closed decomposable hull of a given set $A \subset L_{T}^{2, d}$ (see, e.g., [54] for details). Now, let us consider a special one-dimensional case and the mapping $h: I \times \Omega \rightarrow \mathscr{F}_{c}^{b}\left(R^{1}\right)$ defined by $h(t, \omega):=u$ for every $(t, \omega) \in I \times \Omega$, where $u \in \mathscr{F}_{c}^{b}\left(R^{1}\right)$ is such that $u(z)=$ $(z+1) \rrbracket_{[-1,0)}(z)+(1-z) \rrbracket_{[0,1]}(z)$ for $z \in R^{1}$. Hence $[h(t, \omega)]^{\alpha}=$ $[-1+\alpha, 1-\alpha]$ for $\alpha \in[0,1]$ and $(t, \omega) \in I \times \Omega$. Then by virtue of Corollary 3.9 (as well as Corollary 3.11 and Remark 3.12) in [55] for such chosen mapping $h$, it follows that the set $\operatorname{cl}_{L_{T}^{2,1}} \operatorname{dec}\left(\int_{0}^{T}[h(s)]^{\alpha} d W(s)\right)$ is unbounded as a subset of the space $L_{T}^{2,1}$ for every $\alpha \in[0,1)$. Therefore by (47) for every $\alpha \in[0,1)$ the set $\mathcal{S}_{\mathbf{F}_{T}}^{2}\left(\left[(\mathscr{F}) \int_{0}^{T} h d W\right]^{\alpha}, P\right)$ is unbounded in $L_{T}^{2,1}$ too. Additinaly, by Theorem 3.1 in [56] there exists an $\mathbf{F}_{T^{-}}$ measurable multivalued mapping $H_{T}^{\alpha}: \Omega \rightarrow \mathscr{K}\left(R^{1}\right)$ such that

$$
\mathcal{S}_{\mathbf{F}_{T}}^{2}\left(H_{T}^{\alpha}, P\right)=\mathrm{cl}_{L_{T}^{2,1}} \operatorname{dec}\left(\int_{0}^{T}[h(s)]^{\alpha} d W(s)\right) .
$$

Moreover, due to Theorem 2.2 in [56], one has

$$
\begin{aligned}
& E\left\{\left\|H_{T}^{\alpha} \mid\right\|_{R^{1}}^{2}\right\} \\
& =\sup \left\{E\left(v^{2}\right): v \in \operatorname{cl}_{L_{T}^{2,1}} \operatorname{dec}\left(\int_{0}^{T}[h(s)]^{\alpha} d W(s)\right)\right\}
\end{aligned}
$$


for every $\alpha \in[0,1]$. By (47) and (48) it follows that $H_{T}^{\alpha} \subseteq$ $\left[(\mathscr{F}) \int_{0}^{T} h d W\right]^{\alpha} P$-a.s. for $\alpha \in[0,1]$ and therefore we also have $\left\|\left|H_{T}^{\alpha}\right|\right\|_{R^{1}} \leq\|\|\left[(\mathscr{F}) \int_{0}^{T} h d W\right]^{\alpha} \mid \|_{R^{1}} P$-a.s. Hence, due to unboundedness of the set $\mathrm{cl}_{L_{T}^{2,1}} \operatorname{dec}\left(\int_{0}^{T}[h(s)]^{\alpha} d W(s)\right)$ and one gets $E\left\{\left\|\mid\left[(\mathscr{F}) \int_{0}^{T} h d W\right]^{\alpha}\right\| \|_{R^{1}}^{2}\right\}=+\infty$ for every $\alpha \in[0,1)$.

This analysis shows that it is not true that a natural extension of the notion of fuzzy stochastic Itô's integral, that is, a fuzzy random variable $(\mathscr{F}) \int_{0}^{T} h d W$, is integrably bounded when fuzzy-valued integrands have nonsingle-valued $\alpha$-level sets. Notice also that if we have the equality in (47) then we get the minimal possible (in the sense of inclusion) extension of the notion of the fuzzy stochastic Itô's integral. In this case one has $H_{T}^{\alpha}=\left[(\mathscr{F}) \int_{0}^{T} h d W\right]^{\alpha} P$-a.s. for $\alpha \in[0,1]$, and the whole above analysis can be applied. Let us note also that the same argumentation applies when we choose any point of time $t \epsilon$ $I$ instead of $T$. Another remark is that it is easy to see that due to Corollary 3.9 (as well as Corollary 3.11 and Remark 3.12) in [55]) one can construct many other examples of nontrivial fuzzy integrands for which fuzzy stochastic Itô's integrals are not integrally bounded. This means that the fuzzy stochastic integral equation

$$
\begin{aligned}
x(t)= & x_{0} \oplus(\mathscr{F}) \int_{0}^{t} f(s, x(s)) d s \\
& \oplus(\mathscr{F}) \int_{0}^{t} g(s, x(s)) d W(s) P \text {-a.e., } \quad t \in I
\end{aligned}
$$

may not be defined properly unless the diffusion term is crisp.

\section{Conflict of Interests}

The author declares that there is no conflict of interests regarding the publication of this paper.

\section{References}

[1] M. Guo, X. Xue, and R. Li, "Impulsive functional differential inclusions and fuzzy population models," Fuzzy Sets and Systems, vol. 138, no. 3, pp. 601-615, 2003.

[2] M. Hanss, Applied Fuzzy Arithmetic: An Introduction with Engineering Applications, Springer, Berlin, Germany, 2005.

[3] M. Oberguggenberger and S. Pittschmann, "Differential equations with fuzzy parameters," Mathematical and Computer Modelling of Dynamical Systems, vol. 5, no. 3, pp. 181-202, 1999.

[4] J. Casasnovas and F. Rosselló, "Averaging fuzzy biopolymers," Fuzzy Sets and Systems, vol. 152, no. 1, pp. 139-158, 2005.

[5] M. S. El Naschie, "From experimental quantum optics to quantum gravity via a fuzzy Kähler manifold," Chaos, Solitons \& Fractals, vol. 25, no. 5, pp. 969-977, 2005.

[6] A. Bencsik, B. Bede, J. Tar, and J. Fodor, "Fuzzy differential equations in modeling of hydraulic differential servo cylinders," in Proceedings of the 3rd Romanian-Hungarian Joint Symposium on Applied Computational Intelligence (SACI '06), Timisoara, Romania, 2006.

[7] M. Bröcker and M. Lemmen, "Nonlinear control methods for disturbance rejection on a hydraulically driven flexible robot," in Proceedings of the 2nd International Workshop on Robot Motion and Control (RoMoCo '01), pp. 213-217, Bukowy Dworek, Poland, October 2001.

[8] J. K. Tar, I. J. Rudas, and J. F. Bitó, “Group theoretical approach in using canonical transformations and symplectic geometry in the control of approximately modelled mechanical systems interacting with an unmodelled environment," Robotica, vol. 15, no. 2, pp. 163-179, 1997.

[9] R. P. Agarwal, D. O’Regan, and V. Lakshmikantham, "A stacking theorem approach for fuzzy differential equations," Nonlinear Analysis: Theory, Methods \& Applications, vol. 55, no. 3, pp. 299312, 2003.

[10] J. J. Buckley and T. Feuring, "Fuzzy differential equations," Fuzzy Sets and Systems, vol. 110, no. 1, pp. 43-54, 2000.

[11] P. Diamond and P. Kloeden, Metric Spaces of Fuzzy Sets: Theory and Applications, World Scientific Publishing, Singapore, 1994.

[12] P. Diamond, "Time-dependent differential inclusions, cocycle attractors and fuzzy differential equations," IEEE Transactions on Fuzzy Systems, vol. 7, no. 6, pp. 734-740, 1999.

[13] E. Hüllermeier, "An approach to modelling and simulation of uncertain dynamical systems," International Journal of Uncertainty, Fuzziness and Knowledge-Based Systems, vol. 5, no. 2, pp. 117-137, 1997.

[14] X. Guo and D. Shang, "Approximate solution of ${ }^{n}$ th-order fuzzy linear differential equations," Mathematical Problems in Engineering, vol. 2013, Article ID 406240, 12 pages, 2013.

[15] O. Kaleva, "Fuzzy differential equations," Fuzzy Sets and Systems, vol. 24, no. 3, pp. 301-317, 1987.

[16] O. Kaleva, "The Cauchy problem for fuzzy differential equations," Fuzzy Sets and Systems, vol. 35, no. 3, pp. 389-396, 1990.

[17] O. Kaleva, "A note on fuzzy differential equations," Nonlinear Analysis: Theory, Methods \& Applications, vol. 64, no. 5, pp. 895900, 2006.

[18] V. Laksmikantham, S. Leela, and A. S. Vatsala, "Interconnection between set and fuzzy differential equations," Nonlinear Analysis: Theory, Methods \& Applications, vol. 54, no. 2, pp. 351-360, 2003.

[19] V. Lakshmikantham and R. N. Mohapatra, Theory of Fuzzy Differential Equations and Inclusions, Taylor \& Francis, London, UK, 2003.

[20] V. Lakshmikantham and A. A. Tolstonogov, "Existence and interrelation between set and fuzzy differential equations," Nonlinear Analysis: Theory, Methods \& Applications, vol. 55, no. 3, pp. 255-268, 2003.

[21] J. J. Nieto, R. Rodriguez-Lopez, and D. N. Georgiou, "Fuzzy differential systems under generalized metric spaces approach," Dynamic Systems and Applications, vol. 17, no. 1, pp. 1-24, 2008.

[22] T. Rzeżuchowski and J. Wąsowski, "Differential equations with fuzzy parameters via differential inclusions," Journal of Mathematical Analysis and Applications, vol. 255, no. 1, pp. 177-194, 2001.

[23] D. Shang and X. Guo, "Adams predictor-corrector systems for solving fuzzy differential equations," Mathematical Problems in Engineering, vol. 2013, Article ID 312328, 12 pages, 2013.

[24] M. L. Puri and D. A. Ralescu, "Differentials of fuzzy functions," Journal of Mathematical Analysis and Applications, vol. 91, no. 2, pp. 552-558, 1983.

[25] B. Bede and S. G. Gal, "Generalizations of the differentiability of fuzzy-number-valued functions with applications to fuzzy differential equations," Fuzzy Sets and Systems, vol. 151, no. 3, pp. 581-599, 2005. 
[26] B. Bede and S. G. Gal, "Solutions of fuzzy differential equations based on generalized differentiability," Communications in Mathematical Analysis, vol. 9, no. 2, pp. 22-41, 2010.

[27] B. Bede, I. J. Rudas, and A. L. Bencsik, "First order linear fuzzy differential equations under generalized differentiability," Information Sciences, vol. 177, no. 7, pp. 1648-1662, 2007.

[28] T. Gnana Bhaksar, V. Lakshmikantham, and V. Devi, "Revisiting fuzzy differential equations," Nonlinear Analysis: Theory, Methods \& Applications, vol. 58, no. 3-4, pp. 351-358, 2004.

[29] P. Diamond and P. Watson, "Regularity of solution sets for differential inclusions quasi-concave in a parameter," Applied Mathematics Letters, vol. 13, no. 1, pp. 31-35, 2000.

[30] M. T. Malinowski and M. Michta, "Fuzzy stochastic integral equations," Dynamic Systems and Applications, vol. 19, no. 3-4, pp. 473-493, 2010.

[31] M. T. Malinowski and M. Michta, "Stochastic fuzzy differential equations with an application," Kybernetika, vol. 47, no. 1, pp. 123-143, 2011.

[32] M. Michta, "On set-valued stochastic integrals and fuzzy stochastic equations," Fuzzy Sets and Systems, vol. 177, pp. 1-19, 2011.

[33] M. T. Malinowski, "Strong solutions to stochastic fuzzy differential equations of Itô type," Mathematical and Computer Modelling, vol. 55, no. 3-4, pp. 918-928, 2012.

[34] M. T. Malinowski and R. P. Agarwal, "On solutions to setvalued and fuzzy stochastic differential equations," Journal of the Franklin Institute, vol. 352, no. 8, pp. 3014-3043, 2015.

[35] M. T. Malinowski and M. Michta, "Set-valued stochastic integral equations driven by martingales," Journal of Mathematical Analysis and Applications, vol. 394, no. 1, pp. 30-47, 2012.

[36] M. T. Malinowski, M. Michta, and J. Sobolewska, "Set-valued and fuzzy stochastic differential equations driven by semimartingales," Nonlinear Analysis: Theory, Methods \& Applications, vol. 79, pp. 204-220, 2013.

[37] J. Krasińska and M. Michta, "A note on stochastic inclusions approach for fuzzy stochastic differential equations driven by semimartingales," Dynamic Systems and Applications, vol. 22, no. 4, pp. 503-516, 2013.

[38] M. T. Malinowski, "On a new set-valued stochastic integral with respect to semimartingales and its applications," Journal of Mathematical Analysis and Applications, vol. 408, no. 2, pp. 669-680, 2013.

[39] M. T. Malinowski, "Set-valued and fuzzy stochastic integral equations driven by semimartingales under Osgood condition," Open Mathematics, vol. 13, no. 1, pp. 106-134, 2015.

[40] M. T. Malinowski, "Approximation schemes for fuzzy stochastic integral equations," Applied Mathematics and Computation, vol. 219, no. 24, pp. 11278-11290, 2013.

[41] M. T. Malinowski, "Itô type stochastic fuzzy differential equations with delay," Systems and Control Letters, vol. 61, pp. 692701, 2012.

[42] M. T. Malinowski, "Some properties of strong solutions to stochastic fuzzy differential equations," Information Sciences, vol. 252, pp. 62-80, 2013.

[43] M. T. Malinowski, "Modeling with stochastic fuzzy differential equations," in Mathematics of Uncertainty Modeling in the Analysis of Engineering and Science Problems, pp. 150-172, IGI Global, 2014.

[44] M. T. Malinowski, "Fuzzy and set-valued stochastic differential equations with local Lipschitz condition," IEEE Transactions on Fuzzy Systems, vol. 23, no. 5, pp. 1831-1898, 2014.
[45] P. Protter, Stochastic Integration and Differential Equations: A New Approach, Springer, New York, NY, USA, 1990.

[46] K. L. Chung and R. J. Williams, Introduction to Stochastic Integration, Birkhäuser, Boston, Mass, USA, 1983.

[47] S. Hu and N. S. Papageorgiou, Handbook of Multivalued Analysis, Volume I: Theory, Kluwer Academic Publishers, Boston, Mass, USA, 1997.

[48] D. Qiu and L. Shu, "Supremum metric on the space of fuzzy sets and common fixed point theorems for fuzzy mappings," Information Sciences, vol. 178, no. 18, pp. 3595-3604, 2008.

[49] A. Colubi, J. S. Domínguez-Menchero, M. López-Díaz, and D. Ralescu, "A $D_{E}[0,1]$ representation of random upper semicontinuous functions," Proceedings of the American Mathematical Society, vol. 130, no. 11, pp. 3237-3242, 2002.

[50] M. Stojaković and Z. Stojaković, "Series of fuzzy sets," Fuzzy Sets and Systems, vol. 160, no. 21, pp. 3115-3127, 2009.

[51] C. V. Negoita and D. A. Ralescu, Applications of Fuzzy Sets to Systems Analysis, Wiley, New York, NY, USA, 1975.

[52] M. Kisielewicz, Differential Inclusions and Optimal Control, Kluwer Academic Publishers, Dordrecht, The Netherlands, 1991.

[53] M. Kisielewicz, Stochastic Differential Inclusions and Applications, Springer, New York, NY, USA, 2013.

[54] A. Fryszkowski, Fixed Point Theory for Decomposable Sets, Kluwer Academic Publishers, Dordrecht, The Netherlands, 2004.

[55] M. Michta, "Remarks on unboudedness of set-valued Itô's stochastic integrals," Journal of Mathematical Analysis and Applications, vol. 424, no. 1, pp. 651-663, 2015.

[56] F. Hiai and H. Umegaki, "Integrals, conditional expectations, and martingales of multivalued functions," Journal of Multivariate Analysis, vol. 7, no. 1, pp. 149-182, 1977. 


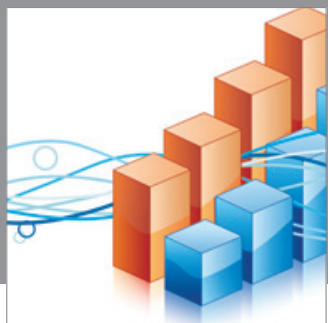

Advances in

Operations Research

mansans

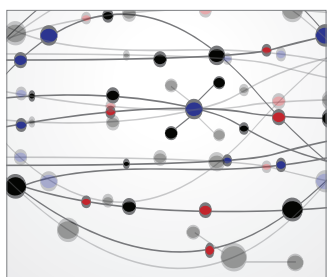

The Scientific World Journal
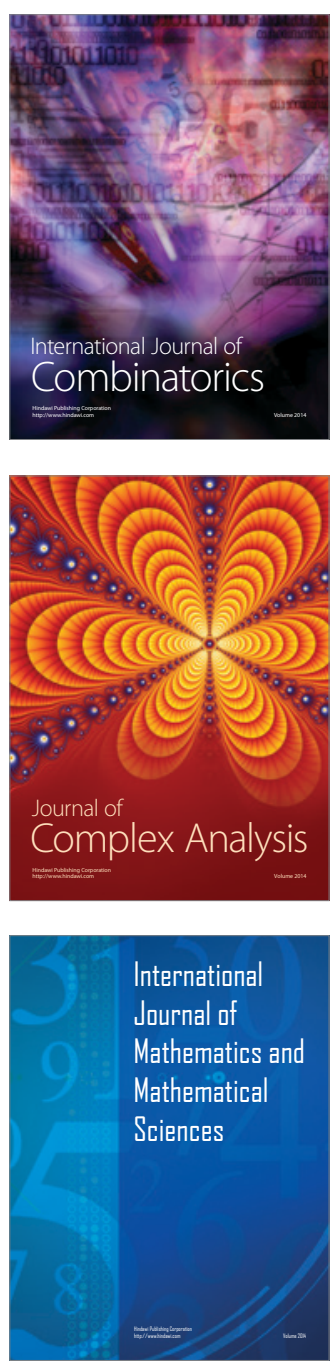
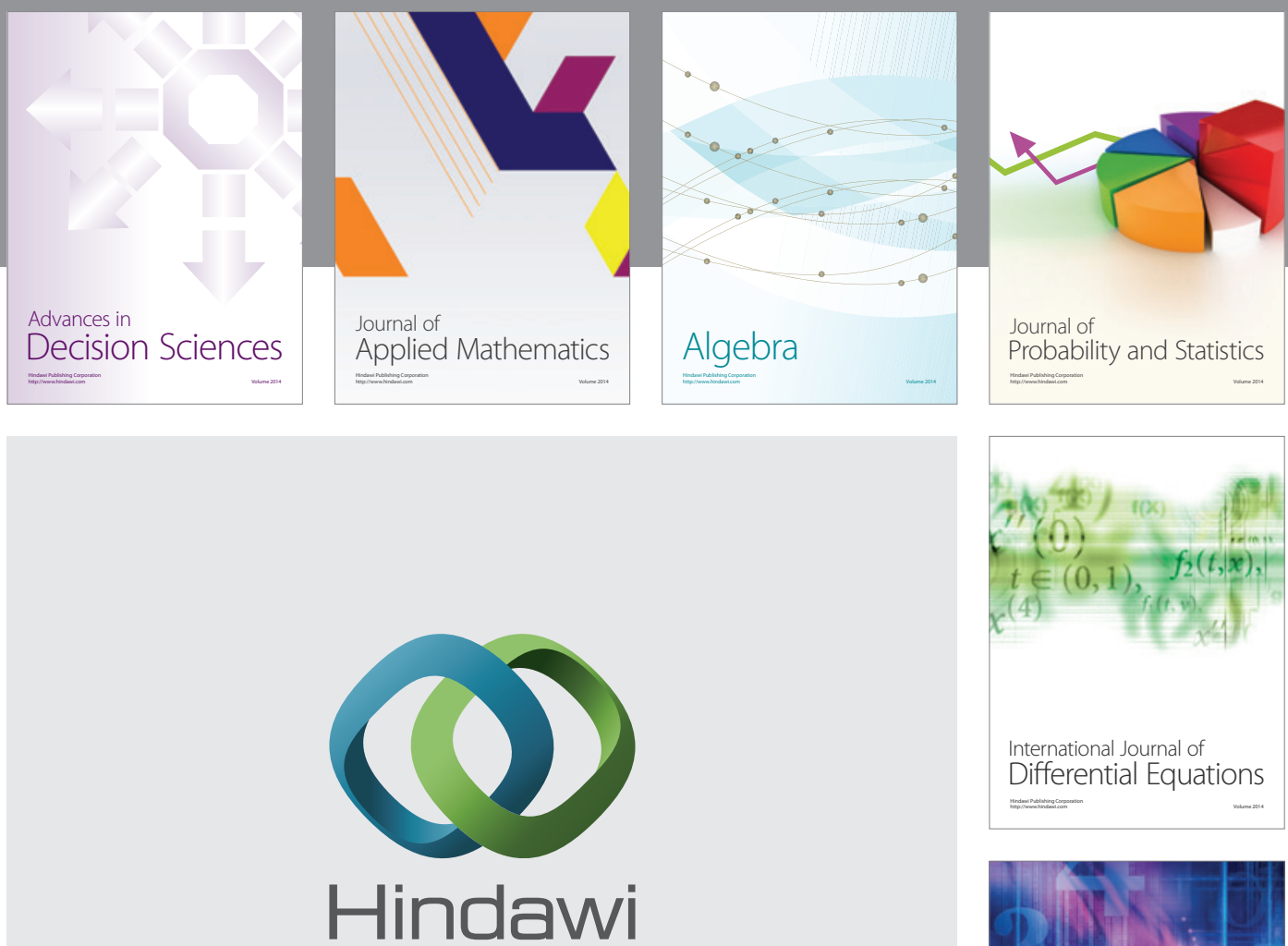

Submit your manuscripts at http://www.hindawi.com
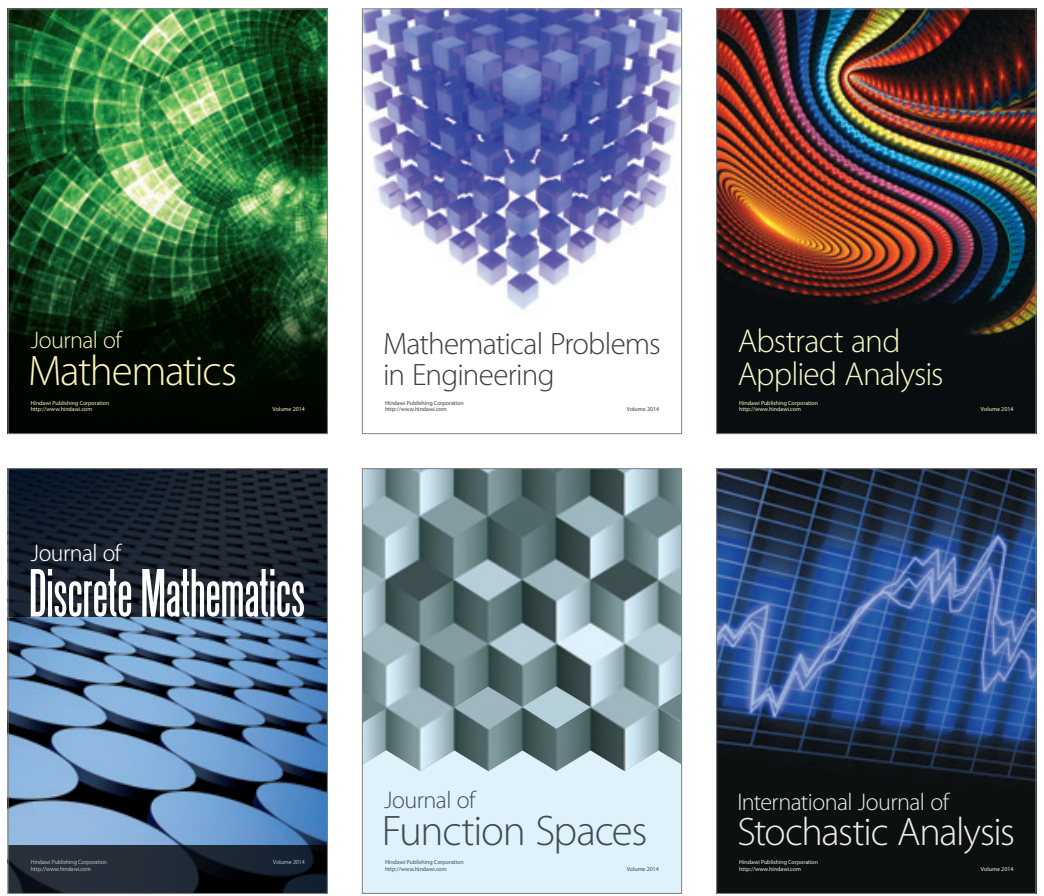

Journal of

Function Spaces

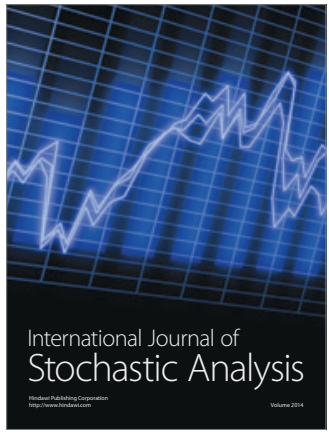

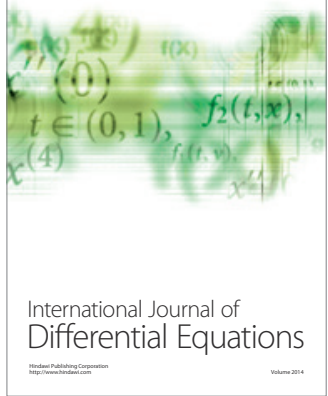
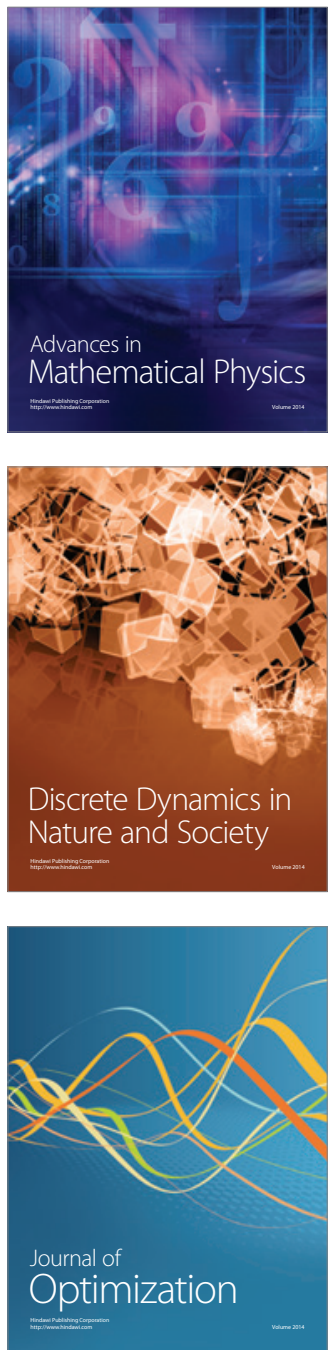